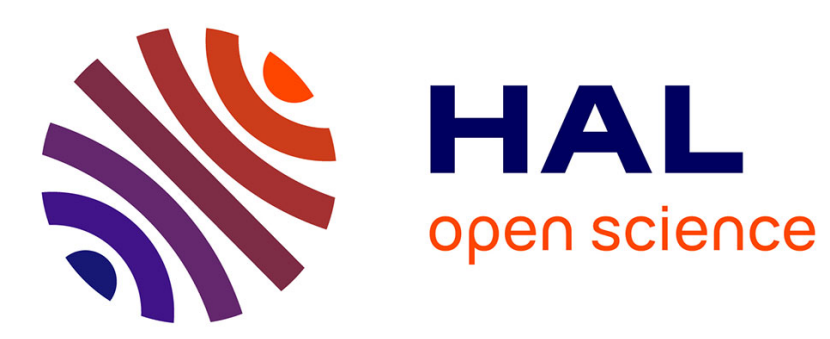

\title{
Some figures of merit so as to compare digital Fresnel holography and speckle interferometry
}

Pierre Slangen, Mayssa Karray, Pascal Picart

\section{To cite this version:}

Pierre Slangen, Mayssa Karray, Pascal Picart. Some figures of merit so as to compare digital Fresnel holography and speckle interferometry. SPIE Optical Metrology, May 2011, Munich, France. pp.808205, 10.1117/12.890865 . hal-02012143

\section{HAL Id: hal-02012143 \\ https://hal.science/hal-02012143}

Submitted on 26 Feb 2020

HAL is a multi-disciplinary open access archive for the deposit and dissemination of scientific research documents, whether they are published or not. The documents may come from teaching and research institutions in France or abroad, or from public or private research centers.
L'archive ouverte pluridisciplinaire $\mathbf{H A L}$, est destinée au dépôt et à la diffusion de documents scientifiques de niveau recherche, publiés ou non, émanant des établissements d'enseignement et de recherche français ou étrangers, des laboratoires publics ou privés. 


\title{
Some figures of merit so as to compare digital Fresnel holography and speckle interferometry
}

\author{
Pierre Slangen*a , Mayssa Karray ${ }^{\mathrm{b}}$, Pascal Picart ${ }^{\mathrm{b}, \mathrm{c}}$ \\ ${ }^{a}$ Ecole des Mines d'Alès, 6 Avenue de Clavières, 30100 ALES, France; \\ ${ }^{b}$ LAUM CNRS, Université du Maine, Av. O. Messiaen, 72085 LE MANS, France \\ ${ }^{c}$ ENSIM-École Nationale Supérieure d’Ingénieurs du Mans, rue Aristote, 72085 LE MANS, France \\ *corresponding author; email: pierre.slangen@mines-ales.fr
}

\begin{abstract}
This paper presents a detailed analysis of figures of merit to compare digital Fresnel holography and speckle interferometry. The analysis is based on both theoretical and experimental analyses. A theoretical analysis of the influence of the aperture and lens in the case of speckle interferometry is developed. Compared to digital Fresnel holography, this element is a critical point influencing Shannon conditions, spatial resolution, spatial filtering and photometric efficiency. Optimal filtering and image recovering conditions are thus established. The theoretical analysis is validated by experimental results. The influence of the speckle decorrelation is estimated for the measurement of mechanical deformations. The same mechanical loading has been applied for both experimental configurations. The probability density of the noise map is then estimated. Fitting the curves along the theoretical analysis results in an objective comparison of the decorrelation degrees, and gives keys to compare the decorrelation sensitivity of the methods.
\end{abstract}

Keywords: speckle interferometry, digital holography, spatial carrier frequency, Fourier transform, Fresnel transform

\section{INTRODUCTION}

Optical techniques using speckle pattern have been widely studied and used for many years ${ }^{1-4}$. In particular these techniques give a fruitful contribution to the analysis of mechanical structures under strain, by providing whole field information on displacement. According to the optical configuration of the experimental set-up, speckle interferometry (SI) (or ESPI-electronic speckle pattern interferometry) ${ }^{5-8}$ can be used to measure the in-plane or out-of-plane displacement of the object under study, or both displacements simultaneously ${ }^{9},{ }^{10}$. SI interferograms can be processed by phase-shifting techniques (either temporal or spatial) ${ }^{11,12}$ or by Fourier transform ${ }^{13-16}$. The latter requires only one recording, whereas the other one requires at least three recordings. Almost at the same time, digital holography was experimentally demonstrated ${ }^{17,18}$. Digital holography (DH) can now be advantageously used to image objects. Lately, many fascinating possibilities have been demonstrated: focusing can be chosen freely ${ }^{19}$, ${ }^{20}$, a single hologram can provide amplitude-contrast and phase-contrast microscopic imaging ${ }^{20}$, image aberrations can be compensated ${ }^{21}$, digital color holography and time-averaging are also possible ${ }^{22-24}$. The processing of digital holograms is generally based on the discrete Fresnel transform ${ }^{17}$, which is applied on a single digital hologram ${ }^{17,24}$ or after a pre-processing based on phase shifting ${ }^{18}$. Note that digital holography exhibits various architectures such as Fresnel holography, Fraunhofer holography, Fourier holography, Lens-less Fourier holography and imageplane holography ${ }^{4}$.

The methods of DH and SI find their interest in contact-less metrology with applications in mechanical strain, vibrations, displacement field or surface shape measurements. There are some strong similarities between both methods, especially concerning data processing. However, some figures of merit explaining the advantages and the drawbacks of the methods have not been discussed in literature. These figures of merit must indicate, most objectively, the differences and the advantages. This paper proposes to analyze both methods on the basis of 5 criteria: filtering and numerical processing, the influence of the aperture, photometric efficiency, spatial resolution and decorrelation noise.

\section{METHODOLOGY}

As discussed in the previous section, processing interferograms can be based on Fourier transformation or on phase-shifting. The latter needs at least 3 recordings to efficiently process the data. Note that a huge amount of literature 
describing SI optical arrangements and processing aspects is available. The distinction appears to be between static and dynamic regimes of object deformations. Dynamic phase shifting can be applied to situations whose ratio frame rate/phase change rate is high ${ }^{25}$. Some other methods exist to study dynamic events, but they are based on modified 2D Hilbert transform ${ }^{26}$ or on correlation techniques ${ }^{27,28}$ and need at least two interferograms to generate the correlation fringes or to remove the zero-order. Of course, pixel temporal history analysis has also been developed ${ }^{29}$ and is not directly based on an image-plane analysis. This paper focuses on the case where only one spatially phase-biased interferogram is recorded ${ }^{13,17,24}$. This means that, for digital Fresnel holography, the configuration is offaxis. This choice is justified as follows: recovering a single interferogram is a powerful tool to study dynamic events but also to carry out high speed acquisition and to process interferograms. Examples demonstrating the suitability of such an approach can be found in ${ }^{30}$ for digital Fresnel holography and ${ }^{31,32}$ for speckle interferometry. As we aim at comparing objectively both methods, the same constraints must be applied. Indeed, the experimental optimization of such methods can be performed according to several degrees of freedom. The amplitude of the reference wave can particularly be increased, compared to that of the object beam, in order to get more flexibility in the Shannon conditions when recording, especially as concerns the non-overlapping of the three diffraction orders ${ }^{14,33}$. Here, we consider that the reference waves of both methods are plane waves and are experimentally adjusted to have the same amplitude. Focus is on the information carried by the object wave when the object is illuminated under the same conditions. The distance between the sensor and the object is fixed. The spatial frequencies of the reference beam are fixed and for speckle interferometry, a lens is added to form the image onto the sensor area. This lens is associated to an iris diaphragm, whose role is to limit the aperture of the beam passing through the imaging system. In $1997^{14}$, G. Pedrini presented the first comparative study between DH and SI and he pointed out that SI is a particular case of DH because the digital reconstructed hologram leads to the simulation of the complex amplitude in the space. Lastly, P. Jacquot discussed on use of speckle interferometry in mechanical engineering ${ }^{34}$. P. Jacquot suggested that only the phase of the recorded pattern in the detector plane is of concern and that this important difference suffices to distinguish the SI and DH techniques unambiguously. G. Pedrini ${ }^{14}$ discussed qualitatively on the object reconstructions and the spatial resolutions of both methods. The approach proposed here aims at taking into account both theoretical and experimental aspects to achieve an objective comparison.

\section{THEORETICAL BACKGROUND}

This section presents the theoretical background of both experimental methods by considering the recording/processing of a unique interferogram. As a general rule, let us consider an extended object, sized $\Delta A_{x} \times \Delta A_{y}$, illuminated by a coherent monochromatic wave with wavelength $\lambda$ and a set of reference coordinates attached to the object $(x, y, z)$ and to the recording plane $(X, Y, z)$. In the next section, we will consider a recording sensor $M \times N$ pixels with pitches $p_{x}=p_{y}$.

\subsection{Digital holography: recording and reconstruction}

In the case of digital holography, the object diffracts a wave to the recording plane, localized at distance $d_{0}$. Figure 1 illustrates the experimental setup and notations.
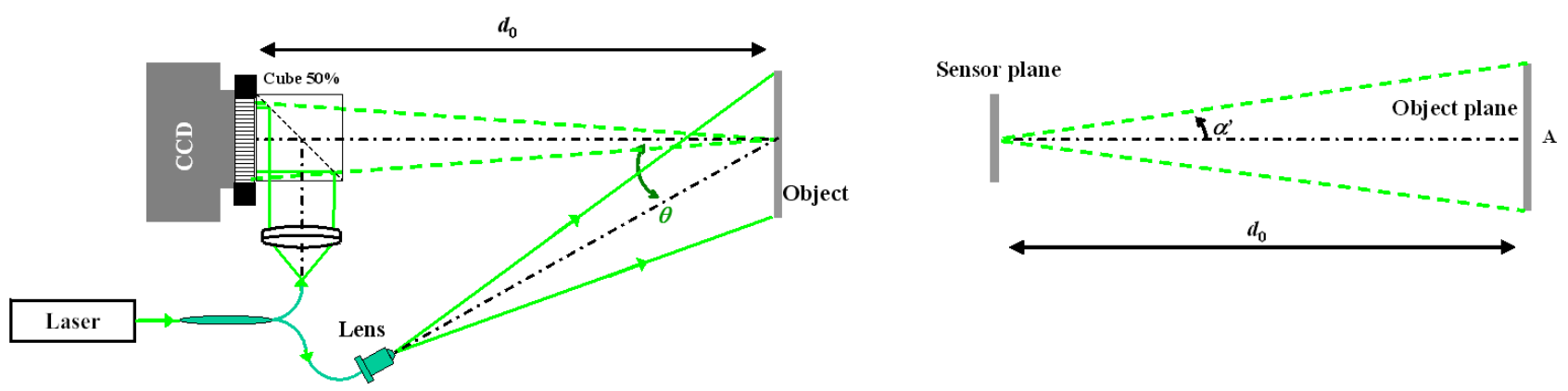

Figure 1. Optical setup for digital Fresnel holography and notations

The object surface generates a wave front that will be noted according to equation 1:

$$
A(x, y)=A_{0}(x, y) \exp \left(i \psi_{0}(x, y)\right)
$$


Amplitude $A_{0}$ describes the object reflectivity and phase $\psi_{0}$ describes its surface or shape $(i=\sqrt{-1})$. Phase $\psi_{0}$ is random and uniformly distributed over the range $]-\pi,+\pi]$. When taking into account the diffraction theory under the Fresnel approximations $^{35}$, the object wave diffracted at distance $d_{0}$ is expressed by the following relation:

$$
\begin{aligned}
& O\left(X, Y, d_{0}\right)=-\frac{i \exp \left(2 i \pi d_{0} / \lambda\right)}{\lambda d_{0}} \exp \left(\frac{i \pi}{\lambda d_{0}}\left(X^{2}+Y^{2}\right)\right) \\
& \quad \times \iint A(x, y) \exp \left(\frac{i \pi}{\lambda d_{0}}\left(x^{2}+y^{2}\right)\right) \exp \left(-\frac{2 i \pi}{\lambda d_{0}}(x X+y Y)\right) d x d y
\end{aligned}
$$

Note that since the object is rough, the diffracted field at distance $d_{0}$ is a speckle field which has a random and uniform phase over the range $]-\pi,+\pi]$. In the Fourier plane, the object wave occupies a spatial frequency bandwidth equal to $(\Delta u \times \Delta v)=\left(\Delta A_{x} / \lambda d_{0} \times \Delta A_{y} / \lambda d_{0}\right)$. In the recording plane, the object wave is mixed with a reference wave written as:

$$
R(x, y)=a_{r} \exp \left(2 i \pi\left(u_{0} x+v_{0} y\right)\right) \text {, }
$$

with $a_{r}$ its modulus and $\left(u_{0}, v_{0}\right)$, the carrier spatial frequencies. In the case where $\left(u_{0}, v_{0}\right) \neq(0,0)$ we get "off-axis digital holography" and when $\left(u_{0}, v_{0}\right)=(0,0)$, we get "in-line digital holography". As pointed out, we consider here the case of "off-axis digital holography". The total intensity received by the recording sensor is the digital hologram:

$$
H=|O|^{2}+|R|^{2}+O R^{*}+O^{*} R \text {. }
$$

The Shannon theorem applied to off-axis digital holography, resulting in the spatial separation of the three diffraction orders appearing in eq. 4 , leads to the optimal recording distance ${ }^{36}$. It is given for a circular object shape with diameter $\Delta A=\Delta A_{x}=\Delta A_{y}$ :

$$
d_{0}=\frac{(2+3 \sqrt{2}) p_{x}}{2 \lambda} \Delta A
$$

Ideally, the spatial frequencies of the reference wave must be adjusted to $\left(u_{0}, v_{0}\right)=\left( \pm 1 /(6+2 \sqrt{ } 2) p_{x}, \pm 1 /(6+2 \sqrt{ } 2) p_{y}\right)$ for the circular object ${ }^{36}$. Practically, the spatial frequencies can be adjusted following this method: the reference beam is perpendicular to the recording plane but the object is laterally shifted by quantities:

$$
\left\{\begin{array}{l}
\Delta X=\frac{\lambda d_{0}}{p_{x}}\left(\frac{1}{2}-\frac{1}{2+3 \sqrt{2}}\right) \\
\Delta Y=\frac{\lambda d_{0}}{p_{y}}\left(\frac{1}{2}-\frac{1}{2+3 \sqrt{2}}\right)
\end{array} .\right.
$$

The reconstructions of the amplitude and the phase of the encoded object are based on the numerical simulation of light diffraction on the numerical aperture included in the digital hologram. For a reconstruction distance equal to $d_{r}=d_{0}$, the reconstructed field $A_{r}$ is given by the discrete version of eq. 2 (known as DFT algorithm) ${ }^{17,19,36,37}$. The +1 order is then localized at spatial coordinates $\left(\lambda d_{0} u_{0}, \lambda d_{0} v_{0}\right)$. The computation leads to complex-valued results, from which the amplitude image (modulus) and the phase image (argument) can be extracted. If the reconstructed plane is computed with $(K, L) \geq(M, N)$ data points, then the sampling pitches in the reconstructed plane are equal to $\Delta \eta=\lambda d_{0} / L p_{x}$ and $\Delta \xi=\lambda d_{0} / K p_{y}{ }^{17,}{ }^{36}$. The discrete Fresnel transform is adapted to a large range of object sizes and shapes. The second possibility to reconstruct the object is based on the convolution formulae of diffraction. An exhaustive description was provided by Kreis in $1997^{37}$. This means that the reconstructed field is obtained by this convolution equation ( $\otimes$ means convolution), at any distance $d_{r}$ :

$$
A_{r}\left(x, y, d_{r}\right)=H(x, y) \otimes h\left(x, y, d_{r}\right),
$$

where $h\left(x, y, d_{r}\right)$ is the kernel associated to diffraction along distance $d_{r}$. The convolution kernel can be the impulse response of the free space propagation. Such a kernel leads to a transfer function, which is the Fourier transform of the impulse response. The mathematical expression of the kernel is given by Goodman ${ }^{35}$ and must be adapted to off-axis holography ${ }^{38}$ : 


$$
h\left(x, y, d_{r}\right)=\frac{i d_{r}}{\lambda} \frac{\exp \left[2 i \pi / \lambda \sqrt{d_{r}^{2}+x^{2}+y^{2}}\right]}{d_{r}^{2}+x^{2}+y^{2}} \exp \left(-2 i \pi\left(u_{0} x+v_{0} y\right)\right),
$$

The angular spectrum transfer function can also be used as the transfer function of the reconstruction process. In this case the mathematical expression is adapted ${ }^{39}$ and given by:

$$
G\left(u, v, d_{r}\right)=\left\{\begin{array}{l}
\exp \left[2 i \pi d_{r} / \lambda \sqrt{1-\lambda^{2}\left(u-u_{0}\right)^{2}-\lambda^{2}\left(v-v_{0}\right)^{2}}\right] \\
\quad \text { if }\left|u-u_{0}\right| \leq L p_{x} / 2 \lambda d_{r} \text { and }\left|v-v_{0}\right| \leq K p_{y} / 2 \lambda d_{r} \\
0 \quad \text { elsewhere }
\end{array}\right.
$$

The reader may look at references ${ }^{38,39}$ for further details on the reconstruction process. The practical computation of such an equation can be performed according to the properties of the Fourier transform, thus leading to double Fourier transform algorithm (DFFT):

$$
A_{r}=F T^{-1}[F T[H] \times F T[h],
$$

which includes three FFT, when using the impulse response, or only two FFT, when using the angular spectrum transfer function:

$$
A_{r}=F T^{-1}[F T[H] \times G],
$$

In the DFFT algorithms the reconstructed object is sampled by a number of data points that can be chosen freely with $(K, L) \geq(M, N)$, whereas with the DFT algorithm, the number of data points is given by $(K, L)=\left(\Delta A_{x} / \Delta \eta ; \Delta A_{y} / \Delta \xi\right)$.

\subsection{Speckle interferometry: recording and reconstruction}

In the case of speckle interferometry, there is an imaging lens associated to a variable aperture close to the lens. In the classical method $^{10,14}$, the aperture is placed at the front focal plane of the lens. However, commercial imaging systems include a circular aperture (iris diaphragm) inside the set of lenses. In this study, we consider the case of commercial lenses for which the aperture is not localized at the focal plane. The aperture has a diameter $\phi_{D}$ and is placed at distance $d_{D}$ from the detector. Figure 2 illustrates the experimental setup. The lens is at position $p$ from the object and the image is at position $p^{\prime}$ from lens.
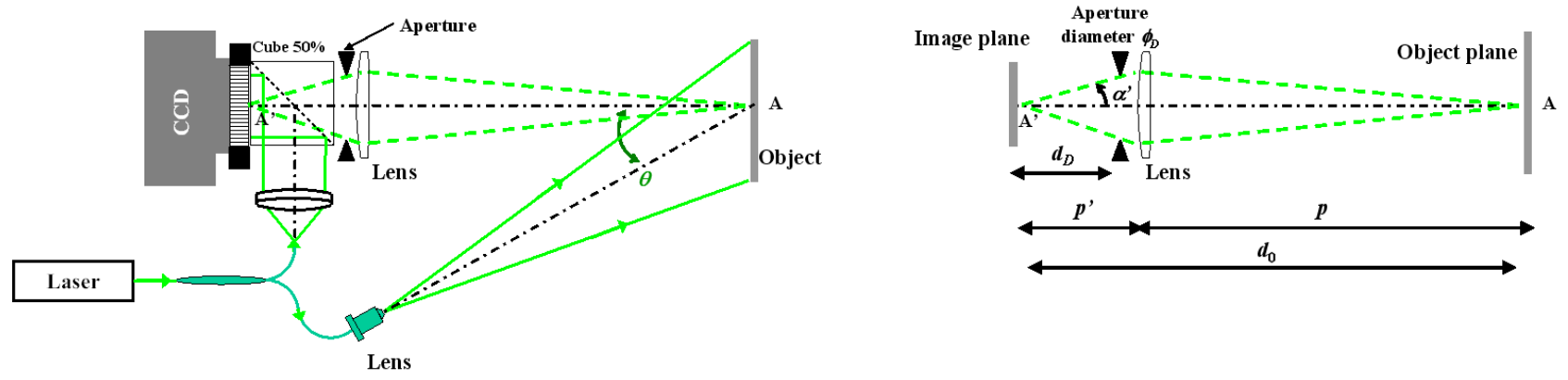

Figure 2. Optical setup for SI and notations

In this case, the object is imaged onto the recording sensor. Let us note $A^{\prime}(x, y)$ the complex field projected onto this plane. In order to optimize the recording, the image of the object must fully lie in the recording plane, so the transverse magnification must be set at $|\gamma|=\min \left(N p_{x} / \Delta A_{x} ; M p_{y} / \Delta A_{y}\right)$. In such a configuration, the object wave occupies a spatial frequency bandwidth equal to $(\Delta u \times \Delta v)=\left(\Delta A_{x} / \lambda p \times \Delta A_{y} / \lambda p\right)=\left(|\gamma| \Delta A_{x} / \lambda p^{\prime} \times|\gamma| \Delta A_{y} / \lambda p^{\prime}\right)$. Now, the recorded specklegram is written:

$$
H=\left|A^{\prime}\right|^{2}+|R|^{2}+A^{\prime} R^{*}+A^{\prime^{*}} R
$$


The reconstruction of the amplitude and phase of the object is performed according to double-FFT strategy, in which the transfer function is a uniform-bandwidth limited function ${ }^{10,13-16}$. In the Fourier plane, the filtering function can thus be written:

$$
G^{\prime}(u, v)= \begin{cases}1 & \text { if }\left|u-u_{0}\right| \leq \Delta A_{x} / 2 \lambda p \text { and }\left|v-v_{0}\right| \leq \Delta A_{y} / 2 \lambda p, \\ 0 & \text { elsewhere }\end{cases}
$$

and the object wave is reconstructed according to:

$$
A^{\prime}{ }_{r}=F T^{-1}\left[F T[H] \times G^{\prime}\right] .
$$

Eq. 14 can be interpreted as a convolution formula similar to eq. 7, but now the impulse response is a two-dimensional sinc function. As can be seen from eq. $(4,9,10,12,13,14)$, there are some strong similarities between both methods, especially concerning data processing. The next section discusses the figures of merit to objectively compare both methods.

\section{FIGURES OF MERIT}

\subsection{Introduction}

The paper proposes a detailed analysis of the 5 figures of merit based on theory and experimental results. We will discuss in particular a theoretical analysis of the influence of the aperture and lens in the case of SI. Compared to digital Fresnel holography, this element is a critical point that influences several aspects of the reconstruction process: filtering/algorithms, aperture, photometric efficiency, spatial resolution and decorrelation noise. Moreover, the conditions for optimal filtering and image recovering are established. We will present a theoretical modeling including all the physical parameters (object albedo, pixel surface, aperture number, transverse magnification, object diameter and laser power) contributing to establishing the photometric ratio as a comparison tool. In order to study the influence of speckle decorrelation, the optical phase from the object reconstruction must be computed. Decorrelation appears when a phase change occurs at the surface of the object. In this paper, we applied a mechanical loading to the object with quite good reproducibility. By varying the amount of loading, different phase changes with different speckle decorrelations are generated. A method based on a low-pass filtering is used to objectively compare the sensitivity of both methods to decorrelation.

Equations given for an objective comparison are explained in the sense of the optimal recording, according to the Shannon conditions. It is obvious that advantages/drawbacks of both methods can be interchanged when experimental conditions deviate from the optimal conditions.

\subsection{Filtering and algorithms}

As previously discussed, the algorithms have some strong similarities. Table 1 gives an overview of the properties of the various reconstruction methods.

Table 1. Attributes of the reconstruction methods

\begin{tabular}{|l|c|c|c|}
\cline { 2 - 4 } \multicolumn{1}{c|}{} & \multicolumn{2}{c|}{ DH } & SI \\
\hline Reconstruction method & Discrete Fresnel transform & Convolution & $\begin{array}{c}\text { Convolution by spectral } \\
\text { filtering }\end{array}$ \\
\hline Number of FFT operations & 1 & 2 or 3 & 2 \\
\hline $\begin{array}{l}\text { Number of data points for } \\
\text { FFT operations }\end{array}$ & free, $(K, L) \geq(M, N)$ & free, $(K, L) \geq(M, N)$ & free, $(K, L) \geq(M, N)$ \\
\hline $\begin{array}{l}\text { Number of data points for } \\
\text { the reconstructed object }\end{array}$ & $\begin{array}{c}\text { imposed, } \\
\text { imp } / \lambda d_{0} \times \Delta A_{y} K p_{y} / \lambda d_{0}\end{array}$ & free, $\geq M \times N$ & $M \times N$ \\
\hline Filtering & $\mathrm{NO}$ & YES & YES \\
\hline Filtering function & - & quadratic phase & binary \\
\hline
\end{tabular}




\begin{tabular}{|l|c|c|c|}
\hline $\begin{array}{l}\text { Needs for an additional } \\
\text { quadratic phase term }\end{array}$ & NO & $\begin{array}{c}\text { YES, for adjustable } \\
\text { magnification }\end{array}$ & NO \\
\hline $\begin{array}{l}\text { Sampling of the } \\
\text { reconstructed object }\end{array}$ & $\left(\lambda d_{0} / L p_{x}, \lambda d_{0} / K p_{y}\right)$ & $\left(p_{x}, p_{y}\right)$ & $\left(p_{x}, p_{y}\right)$ \\
\hline Computation time & $\begin{array}{c}2.703 \mathrm{~s} \text { for } \\
(K, L)=(1024,1360)\end{array}$ & $\begin{array}{c}7.25 \mathrm{~s} \text { for } \\
(K, L)=(1024,1360)\end{array}$ & $\begin{array}{c}3.203 \mathrm{~s} \text { for } \\
(K, L)=(1024,1360)\end{array}$ \\
\hline Degree of complexity & $* *$ & $* * * * *$ & $* * *$ \\
\hline
\end{tabular}

The simplest reconstruction method is the discrete Fresnel transform used in digital holography. The highest complexity is obtained for the convolution method with adjustable magnification but the object can be reconstructed with the same number of data points as the recording sensor ${ }^{39}$. Computation time is given for a PC Pentium 4CPU $2.99 \mathrm{GHz}$ with $2 \mathrm{Go}$ RAM equipped with MATLAB 5.3.

\subsection{Influence of aperture}

This aspect does not clearly appear in literature but it must be pointed out that the specklegram is also the digital hologram of the aperture. This means that speckle interferometry based on the spatial carrier method must be optimized according to the same rules as those for digital Fresnel holography. So, for the digital hologram of the aperture, we must set:

$$
d_{D}=\frac{(2+3 \sqrt{2}) p_{x}}{2 \lambda} \phi_{D}
$$

This means that the numerical aperture of the imaging lens must be set to:

$$
\sin \alpha^{\prime} \cong \frac{\phi_{D}}{2 d_{D}}=\frac{\lambda}{(2+3 \sqrt{2}) p_{x}} .
$$

This point is the key to understand why the spatial carrier method gives results only if the numerical aperture is small. Indeed, if eq. 16 is not fulfilled, the three diffraction orders of the hologram overlap and thus the useful +1 order of eq. 12 is corrupted by the zero order of the digital hologram of the aperture. Note that the numerical aperture only depends on the wavelength and the pixel pitch, whatever the object size may be, since the optimization of the setup is related to the aperture diameter. In the case of digital holography, the useful numerical aperture of the beam is defined according to that of the sensor-to-object beam (Figure 1). It is equal to $\sin \alpha^{\prime} \cong \Delta A / d_{0}=\lambda /(2+3 \sqrt{2}) p_{x}$ thus giving the same result as in eq. 16. From this standpoint, the optimization of the optical setup follows the same rules for both methods and does not depend on the object size.

\subsection{Photometric efficiency}

The circular object is illuminated by the laser, with power $P_{0}$. It is considered as a lambertian diffuser with albedo $R_{d}$. We consider $\tau$ the transmission coefficient of the beam splitter. Then, taking into account that $d_{0}$ must be fixed by eq. 5 and that the object surface is $S_{O}$, the illumination $I_{D H}$ given onto the CCD area for the setup of figure 1 is given by eq. 17:

$$
I_{D H}=\frac{\tau R_{d} \lambda^{2} P_{0}}{(2+3 \sqrt{2})^{2} p_{x}^{2} S_{O}} .
$$

In the case where the object image is projected on the sensor area by the lens, the illumination becomes:

$$
I_{S I}=\frac{\tau R_{d} T_{L} P_{0}}{S_{O}} \sin ^{2} \alpha^{\prime}
$$

where $T_{L}$ is the transmission factor of the imaging lens. According to eq. 16, we have now: 


$$
I_{S I}=\frac{\tau R_{d} \lambda^{2} T_{L} P_{0}}{(2+3 \sqrt{2})^{2} p_{x}^{2} S_{O}},
$$

This leads to the ratio:

$$
\frac{I_{D H}}{I_{S I}}=\frac{1}{T_{L}}>1
$$

So, we can see that the photometric efficiency is slightly in favor of digital holography. This result is also independent from the object size, showing that there is no specific advantage for speckle interferometry for large objects compared to digital holography. From this standpoint and that of sub-section 4.3, an axiom vanishes: speckle interferometry is not "more adapted to large objects than digital holography". Practically, for a large object, it must be placed far from the recording area in the DH method, whereas it can be placed closer in the SI method, by using a lens and an aperture, but in terms of photometric efficiency, a small advantage remains for DH (eq. 20).

\subsection{Spatial resolution}

In digital holography, the spatial resolution in the reconstructed field is $[17,19]$ :

$$
\rho_{x}=\frac{\lambda d_{0}}{N p_{x}} \quad \rho_{y}=\frac{\lambda d_{0}}{M p_{y}} .
$$

Its interpretation is simple: it is the width of a digital diffraction pattern of a rectangular digital aperture with size $\left(N p_{x} \times M p_{y}\right)$ and uniform transmittance. It depends only on the sensor size, wavelength and recording distance. When reconstructing the object by the convolution method with adjustable magnification, the spatial resolution becomes $|\gamma| \rho_{x}$ and $|\not| \rho_{y}$ in the reconstructed horizon, which has a size related to $|\not|[40]$. In the case of speckle interferometry, the spatial resolution is influenced by the imaging lens. The impulse response of the full process is related to the impulse response of the imaging lens. Consequently, the spatial resolution is given by the speckle size in the recording plane. The speckle size is related to the diffraction spot of the aperture of the imaging lens, and is given by ${ }^{1}$ :

$$
\rho_{x}^{\prime}=\rho_{y}^{\prime}=\frac{\lambda d_{D}}{\phi_{D}}=\frac{(2+3 \sqrt{2}) p_{x}}{2} \text {. }
$$

Eqs. 21-22 show that the spatial resolutions are different for both methods. It is admitted that the spatial resolution is related to the average speckle size at the reconstructed object surface ${ }^{14,19,36}$. Numerical comparisons of the speckle sizes are given in section 5.4.

\subsection{Influence of speckle decorrelation}

A limitation of both methods is given by the speckle decorrelation which occurs when the object is deformed. This decorrelation adds a high spatial frequency noise to the useful signal. Because of this influence, the raw phase maps are not directly suitable for visualization or comparison with some theoretical results. Furthermore, the raw phase map must be unwrapped with a robust noise immune algorithm, but alternative methods based on a sin-cos filtering may be used ${ }^{41}$. Speckle decorrelation has been theoretically studied by many authors ${ }^{42-45}$. Important results are summarized in ${ }^{1}$. The probability density function depends on the modulus of the complex coherence factor $|\mu|$. For the optical phase, the second-order probability density of the phase noise is given by eq. 23 , where $\beta=|\mu| \cos (\varepsilon)$ :

$$
p(\varepsilon)=\frac{1-|\mu|}{2 \pi}\left(1-\beta^{2}\right)^{-3 / 2}\left(\beta \sin ^{-1} \beta+\frac{\pi \beta}{2}+\sqrt{1-\beta^{2}}\right) .
$$

Eq. 23 describes the probability of measuring the phase noise $\varepsilon$ in the phase difference between two reconstructions for any loading of the object (mechanic, pneumatic, thermal, acoustic, etc.). The measurement of eq. 23 can be performed according to ${ }^{46}$. The subtraction of the low-pass filtered phase difference from the raw phase difference leads to an estimation of the standard deviation of the noise included in the raw data. If $h_{f}(k, l)$ is the $n \times n$ convolution kernel used for the low-pass filtering, then the standard deviation of the measured noise $\sigma_{\Delta}$ is related to the real noise standard deviation $\sigma_{\varepsilon}$ by eq. 24 : 


$$
\sigma_{\Delta}=\sigma_{\varepsilon} \sqrt{1-2 h(0,0)+\sum_{k=-n l=-n}^{k=+n l=+n}\left|h_{f}(k, l)\right|^{2}} .
$$

Eqs. 23-24 are used to analyze the experimental results in section 5.5.

\section{EXPERIMENTAL RESULTS}

\subsection{Experimental parameters}

The interferometer is based on a Mach Zehnder configuration (not detailed) in which the sensor has 8 bits digitization with $M \times N=1024 \times 1360$ pixels sized $4.65 \mu \mathrm{m} \times 4.65 \mu \mathrm{m}$. The laser is a continuous $\mathrm{HeNe}\left(\lambda=632.8 \mathrm{~nm}, P_{0}=30 \mathrm{~mW}\right)$ and the object is a mechanical structure sized $\Delta A_{x} \times \Delta A_{y}=40 \times 35 \mathrm{~mm}^{2}$. The object is localized at $d_{0}=1030 \mathrm{~mm}$ from the sensor area and is illuminated with a circular spot $40 \mathrm{~mm}$ in diameter. In the case of SI, there is an imaging lens associated to a variable aperture close to the lens. The magnification is such that the image of the object entirely covers the CCD area. The spatial frequencies are adjusted according to eq 6 . In order to compare both methods, the digital hologram is reconstructed using the discrete Fresnel transform with $(K, L)=(2048,2048)$ and with the convolution method so that $(K, L)=(M, N)=(1024,1360)$. In the latter case, we get a reconstructed object having the same horizon as that obtained with speckle interferometry (see Table 1). The magnification for the DH convolution method is the same as the physical one obtained with the lens ${ }^{38,39}$, that is $|\gamma|=0.146$. The lens has a focal length of $150 \mathrm{~mm}$ and the aperture is placed at $145 \mathrm{~mm}$ from the sensor. Eq. 15 implies that the optimal diameter in the Shannon sense is $\phi_{D}=6.32 \mathrm{~mm}$, sin $\alpha^{\prime}=0.021$, leading to a $f \#$ equal to 23.8. Since the aperture is an iris diaphragm, the diameter is changed with 4 values $\phi_{D}=\{3.56 ; 5.5 ; 7.41 ; 9.94\} \mathrm{mm}$. Unfortunately, it was not possible to adjust the diaphragm to its optimal diameter. The amplitude of the reference and object beams are adjusted at the same level for both methods. In order to investigate decorrelation, we applied a mechanical loading to the object with a quite good reproducibility. This good reproducibility can be appreciated on experimental results. For each experimental configuration, we recorded 5 states of the object corresponding to 4 mechanical loadings. Raw phase maps are filtered by a moving-average filter sized $5 \times 5$ pixels $^{41}$. The moving-average filter used to study the decorrelation noise according to the method described in ${ }^{46}$ is sized $n \times n=7 \times 7$ pixels, leading to $\sigma_{\varepsilon}=\sigma_{\Delta} / 0.989$.

\subsection{Object reconstructions}

Figure 3 shows the object reconstructed with the three algorithms of Table 1. The exposure time is set to $573 \mathrm{~ms}$. Fig $3 \mathrm{a}$ shows the full reconstructed field exhibiting the three diffraction orders. The useful part of the field of view corresponding to the reconstructed object is sampled by $511 \times 511$ pixels with pitches $\Delta \eta=\Delta \xi=68.44 \mu \mathrm{m}$. Reconstructions of Figures $3 \mathrm{~b}, 3 \mathrm{c}$ are sampled by $M \times N=1024 \times 1360$ pixel with pitches $p_{x}=p_{y}=4.65 \mu \mathrm{m}$.

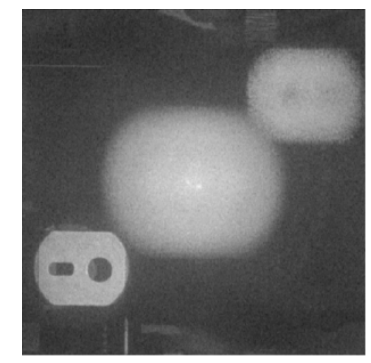

a)

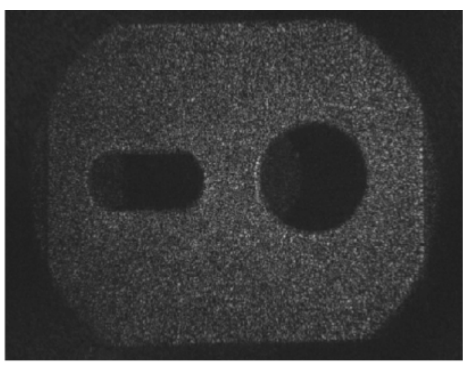

b)

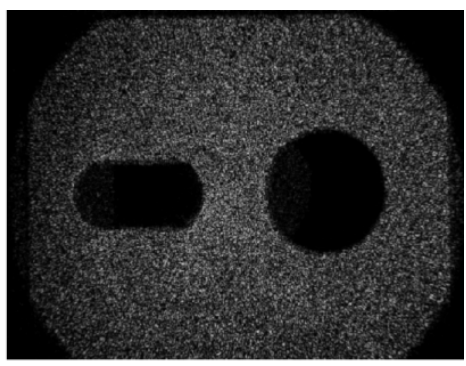

c)

Figure 3. Object reconstructions, a) DH: discrete Fresnel transform, b) DH: convolution with adjustable magnification, c) SI

The image amplitude obtained in Figure $3 \mathrm{~b}$ is quite similar to that of Figure 3c. Figure 4 shows phase changes obtained for DH and SI. Figure $4 \mathrm{a}$ and $4 \mathrm{c}$ show the raw phase map and the filtered one for DH, according to ${ }^{41}$. Figure $4 \mathrm{~b}$ and $4 \mathrm{~d}$ show the raw phase map and the filtered one for SI with $\phi_{D}=5.5 \mathrm{~mm}$. Figure 4 exhibits the very good agreement between both methods and gives appreciation of the correct reproducibility of the mechanical loading. 


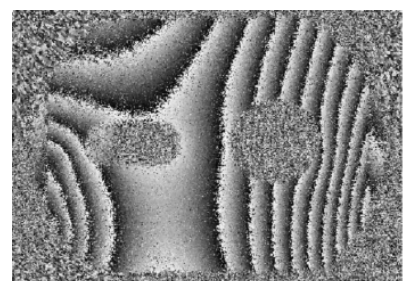

a)

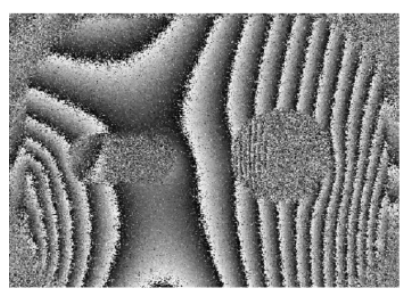

b)
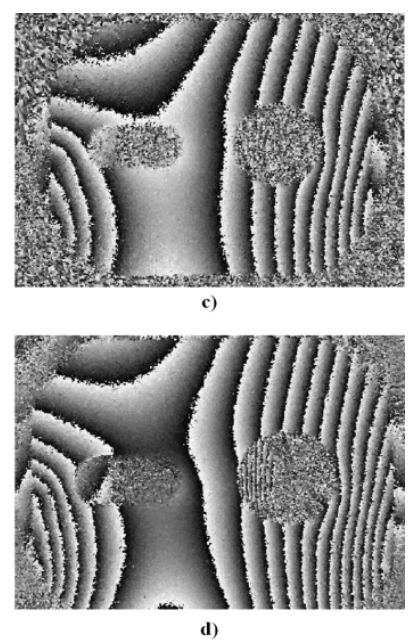

Figure 4. Phase differences, a) DH: raw with convolution, b) SI: raw , c) DH: filtered and d) SI: filtered

\subsection{Influence of aperture}

As pointed out, the specklegram is also the digital hologram of the aperture. So the aperture can be reconstructed from the specklegram by computing the discrete Fresnel transform with $d_{r}=-d_{D}=-145 \mathrm{~mm}$. When $\phi_{D}>6.3 \mathrm{~mm}$, the diameter does not respect the Shannon condition, thus the overlapping of the diffraction orders of the aperture occurs. This means that the useful spectral part of the object, also localized at spatial frequencies $\left(u_{0}, v_{0}\right)$ is overlapped by the contribution of the zero-order of the aperture. Consequently, the phase changes between two mechanical loadings are corrupted and the fringe visibility decreases. Figures $5 \mathrm{a}, \mathrm{b}, \mathrm{c}, \mathrm{d}$ show the reconstructed field with the discrete Fresnel transform and with focus on the iris diaphragm for respectively $\phi_{D}=\{3.56 ; 5.5 ; 7.41 ; 9.94\} \mathrm{mm}$. The image of the aperture can be seen in the bottom left-hand corner of each sub-image. The overlapping can be clearly observed for $\phi_{D} \geq 7.41 \mathrm{~mm}$. Figures $5 \mathrm{e}, \mathrm{f}, \mathrm{g}, \mathrm{h}$ show the phase changes obtained with the four different aperture diameters. The fringe visibility obviously decreases for $\phi_{D} \geq 7.41 \mathrm{~mm}$, the right part of the figure being first affected. Figure $5 \mathrm{~h}$ exhibits the strong visibility decrease obtained for $\phi_{D}=9.94 \mathrm{~mm}$.

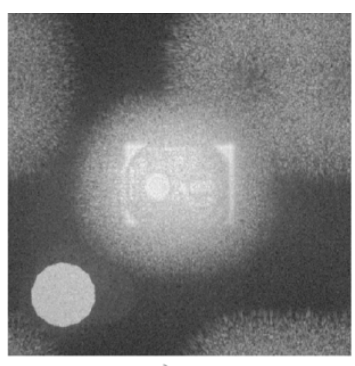

a)

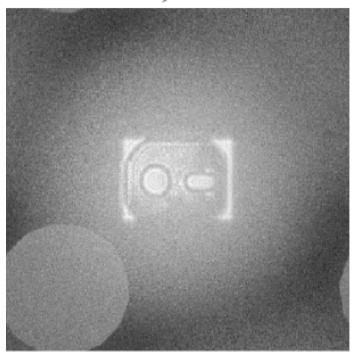

c)

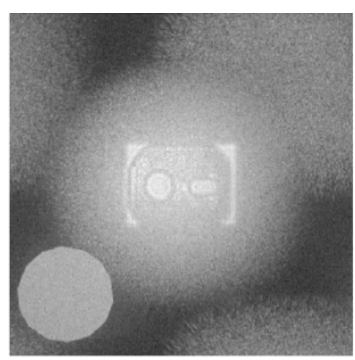

b)

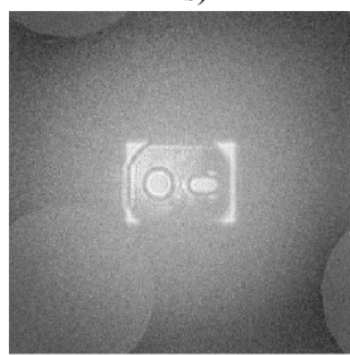

d)

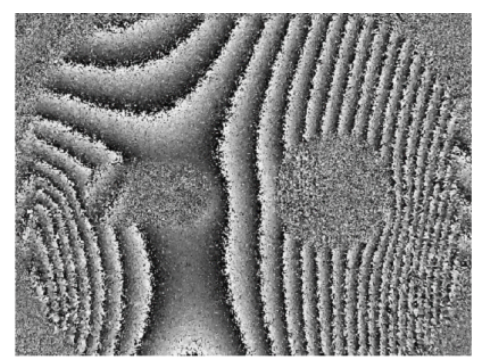

e)

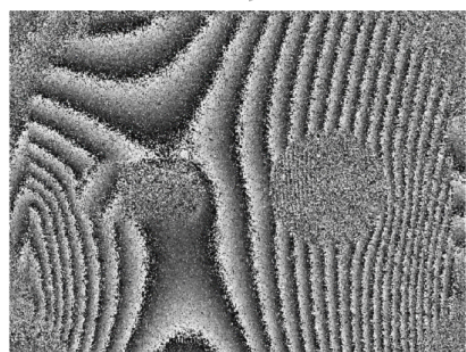

g)

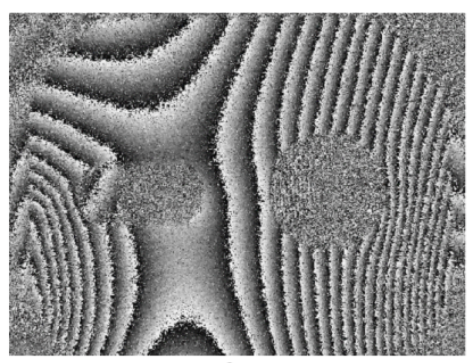

f)

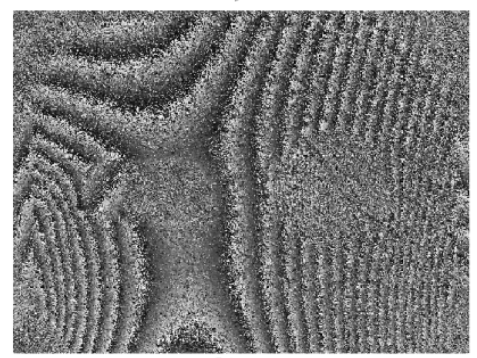

h)

Figure 5. Holographic reconstructions of the aperture a) $\phi_{D}=3.56 \mathrm{~mm}$, b) $\phi_{D}=5.5 \mathrm{~mm}$, c) $\phi_{D}=7.41 \mathrm{~mm}$, d) $\phi_{D}=9.94 \mathrm{~mm}$ and phase differences obtained with SI e) $\left.\left.\left.\phi_{D}=3.56 \mathrm{~mm}, \mathrm{f}\right) \phi_{D}=5.5 \mathrm{~mm}, \mathrm{~g}\right) \phi_{D}=7.41 \mathrm{~mm}, \mathrm{~h}\right) \phi_{D}=9.94 \mathrm{~mm}$ 
This experimental analysis confirms that the method of speckle interferometry with the Fourier-transform evaluation of phase data in spatially phase-biased specklegrams is only valid if the numerical aperture of the imaging system is small, typically with a f\# greater than 20 .

\subsection{Spatial resolution}

From sub-section 4.5 and experimental parameters, the spatial resolutions are $|\gamma| \rho_{x}=15.03 \mu \mathrm{m}$ and $\rho_{y}=19.85 \mu \mathrm{m}$ for DH and $\rho_{x}^{\prime}=\rho_{y}^{\prime}=15.51 \mu \mathrm{m}$ for SI. The values are close, which means the spatial resolutions are the same when fulfilling the Shannon conditions. Experimental measurements are performed as follows: a square zone with $101 \times 101$ pixels is extracted from reconstructed objects and includes sufficient speckle grains. Then the autocorrelation function is computed by FFT algorithms and after normalization $x$-profiles are extracted. Figure 6 shows the $x$-profiles of the autocorrelation functions obtained for DH and the various diameters of the aperture in case of SI.

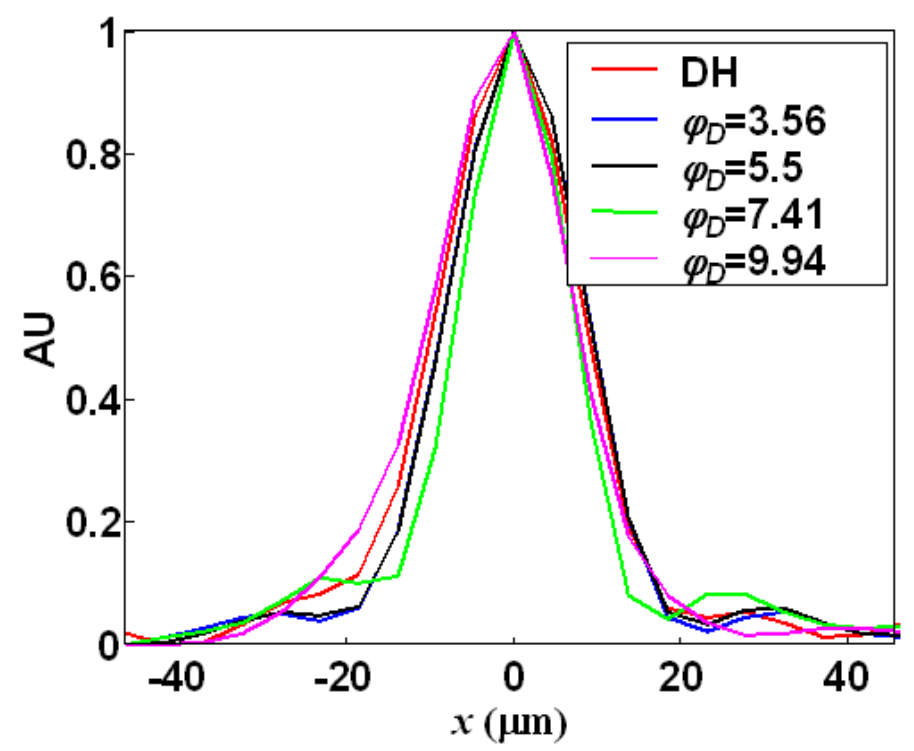

Figure 6. Profiles of autocorrelation functions for DH and SI

Figure 6 shows that spatial resolutions are comparable for all cases. It is slightly better for $\phi_{D}=7.41 \mathrm{~mm}$ since the autocorrelation is narrower, but for $\phi_{D}=9.94 \mathrm{~mm}$, the resolution is degraded since the curve is wider than the theoretical speckle size $(9.23 \mu \mathrm{m})$.

\subsection{Influence of speckle decorrelation}

As discussed previously, the influence of the speckle decorrelation is estimated for the measurement of mechanical deformations. Therefore, we have applied the same mechanical loading in both experimental configurations and then estimated the probability density of the noise maps. We applied four mechanical loadings increased in constant steps. Fitting the curve according to eq. 23 results in an objective comparison of the decorrelation degrees, and it gives keys to compare the decorrelation sensitivity of the methods. Figure 7 shows the probability density function of speckle decorrelation for both DH and SI and for two sates of deformations (1) and (2). State (1) corresponds to the deformation between the $3^{\text {rd }}$ and the $1^{\text {st }}$ recording and state (2) corresponds to the deformation between the $5^{\text {th }}$ and the $1^{\text {st }}$ recording. The estimated value of $|\mu|$ and the noise standard deviation $\sigma_{\varepsilon}$ are indicated for each curve.

Figure 7 shows that the speckle decorrelation is strongly influenced by the aperture size. This result is well known ${ }^{43}$ and is experimentally evidenced by these results. Consequently, DH is less sensitive to decorrelation than SI, if the numerical aperture is greater than $\lambda /(2+3 \sqrt{2}) p_{x}$ (Eq. 16 not fulfilled). In the opposite case, $\sin \alpha^{\prime}<\lambda /(2+3 \sqrt{2}) p_{x}$, SI is less sensitive than $\mathrm{DH}$, but the drawback is that the photometric efficiency is degraded. When the Shannon conditions are fulfilled, $\sin \alpha^{\prime}=\lambda /(2+3 \sqrt{2}) p_{x}$, both methods have the same sensitivity to speckle decorrelation. This can be appreciated in Figure 7 with $\phi_{D}=5.5 \mathrm{~mm}$ for which both DH and SI curves are quasi superposed. For $\phi_{D}=6.3 \mathrm{~mm}$ (Shannon conditions for the aperture) the curves may be expected to be perfectly superposed. 

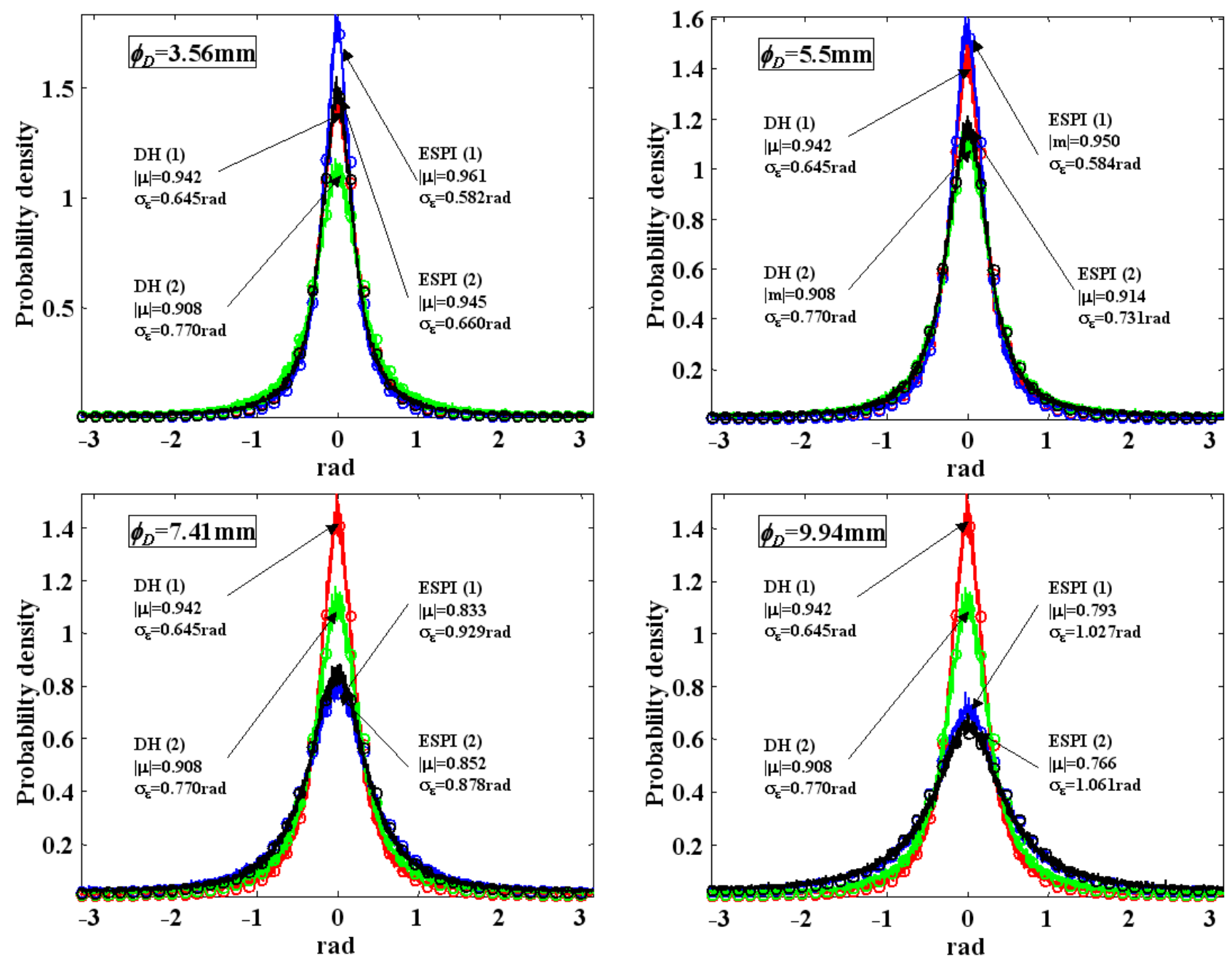

Figure 7. Sensitivity to decorrelation, (1) deformation between the $3^{\text {rd }}$ and the $1^{\text {st }}$ recording, (2) deformation between the $5^{\text {th }}$ and the $1^{\text {st }}$ recording,

\section{CONCLUSION}

This paper exposes the figures of merit to compare digital Fresnel holography and speckle interferometry. These figures of merit include several aspects of the reconstruction process: filtering/algorithms, the influence of the aperture, photometric efficiency, spatial resolution and decorrelation noise. The conditions for optimal filtering and image recovering are established according to the Shannon conditions. The role of the aperture is crucial since the specklegram is its digital hologram and must fulfill the Shannon conditions. The analysis presents theoretical and experimental results that confirm that digital holography and speckle interferometry are close methods giving similar results. Because of the simplicity of the Fresnel transform, digital holography is quite more adapted to simple and automated image processing. The influence of the speckle decorrelation is estimated for the measurement of mechanical deformations. When discarding from Shannon conditions and increasing the aperture diameter, SI suffers from zero-order overlapping and is much more sensitive to decorrelation without increasing the spatial resolution. However, the advantages of using the lens associated to the aperture are related to the photometric efficiency, which can be increased.

As a conclusion, SI is a particular configuration of $\mathrm{DH}$, but like digital holography, speckle interferometry can also be used to determine the complex amplitude and then simulate it in space. 
Further developments will compare both methods in the case of temporal phase shifting for steady state applications.

\section{ACKNOWLEDGEMENT}

The authors are grateful to Pierre Jacquot (EPFL, Lausanne, Switzerland) for very helpful discussions.

\section{REFERENCES}

[1] Dainty, J.C., [Laser Speckle and Related Phenomena], Springer Verlag, Berlin (1984).

[2] West, C. M., [Holographic Interferometry], John Wiley \& Sons, New York (1979).

[3] Jones, R., Wykes, C., [Holographic and Speckle Interferometry], Cambridge University Press, Cambridge (1989).

[4] Kreis, Th., [Holographic Interferometry - Principles and Methods], Akademie Verlag Series in Optical Metrology 1, Akademie Verlag Gmbh, Berlin, (1996).

[5] Jacquot, P., Fournier, J.-M., (Eds.), [Interferometry in Speckle Light: Theory and Applications], Springer-Verlag, Lausanne (2000).

[6] Gastinger, K., Lokberg, O.J., Winter, S., (Eds.), [Speckle Metrology 2003], Proc. SPIE 4933 (2003).

[7] Slangen, P., Cerruti, C., (Eds.), [Speckle06-Speckles, from Grains to Flowers], Proc. SPIE 6341 (2006).

[8] Albertazzi, A., Kaufmann, G.H., (Eds.), [Speckle 2010-Optical metrology], Proc. SPIE 7387 (2010).

[9] Slangen, P., De Veuster, C., Renotte, Y., Berwart, L., Lion, Y., "Computer-aided interferometric measurements of drift and phase shifter calibration for digital speckle pattern interferometry", Opt. Eng. 34(12), 3526-3530 (1995).

[10] Schedin, S., Pedrini, G., Tiziani, H. J., Santoyo, F. M., "Simultaneous three-dimensional dynamic deformation measurements with pulsed digital holography", Appl. Opt. 38(34), 7056-7062 (1999).

[11] Creath, K., "Phase shifting speckle interferometry", Appl. Opt. 24(18), 3053-3058 (1985).

[12] Pedrini, G., Tiziani, H., "Double-pulse electronic speckle interferometry for vibration analysis", Appl. Opt. 33(34), 7857-7863 (1994).

[13] Saldner, H. O., Molin, N. E., Stetson, K. A., "Fourier-transform evaluation of phase data in spatially phase-biased TV holograms", Appl. Opt. 35(2), 332-336 (1996).

[14] Pedrini, G., Tiziani, H., and Zou, Y., "Digital double pulse-TV holography", Opt. Laser Eng. 26, 199-219 (1997).

[15] Pedrini, G., Tiziani, H., "Quantitative evaluation of two-dimensional dynamic deformations using digital holography", Opt. Laser Technol. 29, 249-256 (1997).

[16] Pedrini, G., Froening, Ph., Fessler, H., and Tiziani, H., "Transient vibration measurements using multipulse digital holography", Opt. Laser Technol. 29, 505-511 (1997).

[17] Schnars, U., Jüptner, W., "Direct recording of holograms by a CCD target and numerical reconstruction", Appl.Opt. 33(2), 179-181 (1994).

[18] Yamaguchi, I., Zhang, T., "Phase shifting digital holography", Optics Letters 22, 1268-1270 (1997).

[19] Yamaguchi, I., Kato, J., Ohta, S., Mizuno, J., "Image formation in phase shifting digital holography and application to microscopy", Appl. Opt. 40(34), 6177-6186 (2001).

[20] Cuche, E., Bevilacqua, F., Depeursinge, C., "Digital holography for quantitative phase contrast imaging", Optics Letters 24(5), 291-293 (1999).

[21] De Nicola, S., Ferraro, P., Finizio, A., Pierattin, G., "Correct-image reconstruction in the presence of severe anamorphism by mean of digital holography", Optics Letters 26(13), 974-976 (2001).

[22] Yamaguchi, I., Matsumura, T., Kato, J., "Phase shifting color digital holography", Optics Letters 27(13), 1108-1110 (2002).

[23] Tankam, P., Song, Q., Karray, M., Li, J.C., Desse ,J.M., Picart, P., "Real-time three-sensitivity measurements based on three-color digital Fresnel holographic interferometry", Optics Letters, 35(12), 2055-2057 (2010).

[24] Picart, P., Leval, J., Mounier, D., Gougeon, S., "Time-averaged digital holography", Optics Letters 28(20), 19001902 (2003).

[25] Colonna de Lega, X.C., Jacquot, P., "Deformation measurement with object-induced dynamic phase shifting", Appl. Opt. 35(25), 5115-5121 (1996).

[26] Larkin, K.G., Bone, D.J., Oldfield, M.A., "Natural demodulation of two-dimensional fringe patterns. I. General background of the spiral phase quadrature transform", JOSA A 18(8), 1862-1870 (2001).

[27] Servin, M., Marroquin, J.L., Quiroga, J.A., "Regularized quadrature and phase tracking from a single closed-fringe interferogram", JOSA A 21(3), 411-419 (2004). 
[28] Robin, E. Valle, V., "Phase demodulation from a single fringe pattern based on a correlation technique", Applied Optics 43(22), 4355-4361 (2004).

[29] Equis, S., Jacquot, P., "The empirical mode decomposition: a must-have tool in speckle interferometry?", Optics Express, 17(2), 611-623 (2009).

[30] Picart, P., Leval, J., Piquet, F., Boileau, J.-P., Guimezanes, Th., Dalmont, J.-P., "Tracking high amplitude autooscillations with digital Fresnel holograms", Optics Express, 15(13), 8263-8274 (2007)

[31] Aguayo, D., D., Mendoza Santoyo, F., De la Torre-I., M. H., Salas-Araiza, M. D, Caloca-Mendez, C., Gutierrez Hernandez, D. A., "Insect wing deformation measurements using high speed digital holographic interferometry", Optics Express, 18(6), 5661-5667 (2010).

[32] Pérez-López, C., De la Torre-Ibarra, M. H., Mendoza Santoyo, F., "Very high speed cw digital holographic interferometry", Optics Express, 14(21), 9709-9715 (2006).

[33] Doval, Á. F., Trillo, C., "Hybrid opto-numerical quasi Fourier transform digital holographic camera", Proc. SPIE 6341, 63410Z, (2006).

[34] Jacquot, P., "Speckle interferometry: A review of the principal methods in use for experimental mechanics applications", Strain 44(1), 57-69 (2008).

[35] Goodman, J.W., [Introduction to Fourier Optics (Second Edition),] McGraw-Hill Editions, New York, (1996).

[36] Picart, P., Leval, J., "General theoretical formulation of image formation in digital Fresnel holography", JOSA A, 25, 1744-1761 (2008).

[37] Kreis, Th., Adams, M., Jüptner, W., "Methods of digital holography: a comparison", Proc. SPIE 3098, 224-233 (1997).

[38] Picart, P., Tankam, P., Mounier, D., Peng, Z., Li, J.C, "Spatial bandwidth extended reconstruction for digital color Fresnel holograms", Optics Express 17(11), 9145-9156 (2009).

[39] Li, J.C., Tankam, P., Peng, Z., Picart, P., "Digital holographic reconstruction of large objects using a convolution approach and adjustable magnification", Optics Letters, 34(5), 572-574 (2009).

[40] Tankam, P., Picart, P., Mounier, D., Desse, J.M., Li, J.C, "Method of digital holographic recording and reconstruction using a stacked color image sensor", Applied Optics 49(4), 320-328 (2010).

[41] Aebischer, H.A., Waldner, S., "A simple and effective method for filtering speckle-interferometric phase fringe patterns", Optics Communications, 162(4-6), 205-210 (1999).

[42] Owner-Petersen, P., "Decorrelation and fringe visibility: on the limiting behavior of various electronic specklepattern correlation interferometers", JOSA A 8(7), 1082-1089 (1991).

[43] Lehmann, M., "Decorrelation-induced phase errors in Phase Shifting Speckle Interferometry", Appl. Opt., 36(16), 3657-3667 (1997).

[44]Lehmann, M., "Phase-shifting speckle interferometry with unresolved speckles: A theoretical investigation", Optics Comm. 128(4-6), 325-340 (1996).

[45] Lehmann, M., "Optimization of wave-field intensities in phase-shifting speckle interferometry", Optics Comm. 118(3-4), 199-206 (1995).

[46] Picart, P., Mercier, R., Lamare, M., Breteau, J.-M., "A simple method for measuring the random variation of an interferometer", Measurement Science and Technology 12, 1311-1317 (2001). 